\title{
A Design Method for FES Bone Health Therapy in SCI
}

\author{
Brian Andrews (1,2), James Shippen (3), Monica Armengol (1), Robin Gibbons (4), \\ William Holderbaum (5), William Harwin (1)
}

(1) Department of Bio-Engineering at the University of Reading, UK; (2) The Nuffield Department of Surgical Sciences, Oxford; (3) Department of Industrial Design, Coventry University, UK; (4) Aspire CREATe group University College London, UK; (5) Science and Engineering, Manchester Metropolitan University, UK.

This article is distributed under the terms of the Creative Commons Attribution Noncommercial License (CC BY-NC 4.0) which permits any noncommercial use, distribution, and reproduction in any medium, provided the original author(s) and source are credited.

\begin{abstract}
FES assisted activities such as standing, walking, cycling and rowing induce forces within the leg bones and have been proposed to reduce osteoporosis in spinal cord injury (SCI). However, details of the applied mechanical stimulus for osteogenesis is often not reported. Typically, comparisons of bone density results are made after costly and time consuming clinical trials. These studies have produced inconsistent results and are subject to sample size variations. Here we propose a design process that may be used to predict the clinical outcome based on biomechanical simulation and mechano-biology. This method may allow candidate therapies to be optimized and quantitatively compared. To illustrate the approach we have used data obtained from a rower with complete paraplegia using the RowStim (III) system.
\end{abstract}

Key Words: FES, spinal cord injury, osteoporosis, bone stress, FES rowing

Eur J Transl Myol 26 (4): 297-300

The USA Model Spinal Cord Injury System report fracture incidences due to osteoporosis of $14 \%$ at 5 years, $28 \%$ at 10 years, and $39 \%$ at 15 years post injury, based on outpatient studies, and a prevalence of 25 $46 \%$. The causes of fractures are often unknown or are associated with relatively low energy trauma and have an associated $78 \%$ increase in mortality risk. ${ }^{1}$ The repetitive pattern of limb loading is generally accepted as a major mechanical stimulus determining BMD as illustrated by the Stanford bone mineral density index (BDI) $\quad \mathrm{BDI}=\left(n \cdot[\beta . \mathrm{GRF}]^{m}\right)^{1 /(2 m)}$ where $n$ is the number of loadings per day, $\beta$ is a subject-specific scale factor accounting for differences in body weight, GRF is the ground reaction force, $m$ is an empirical exponent that can be thought of as weighting factor for the relative importance of load magnitude and the number of daily loading cycles. ${ }^{2,3}$ Dudley-Javoroski and Shields (2008) clinically determined that FES ankle plantar-flexion contractions (30 contractions per min with about 8,000 per month for 3yrs) resulted in compressive loads approximately 1.5 times $\mathrm{BW}$ were required to attenuate BMD loss in SCI. ${ }^{4}$ Previous studies indicate modest BMD gains that appear localized to regions of expected higher bone stress. ${ }^{5-9}$ However, it is important to know the 3D distribution of bone mineral such as can be obtained clinically using imaging techniques such as pQCT or high resolution pQCT. ${ }^{6}$ In 1964 Harold Frost proposed a regulatory mechanism for bone adaptation to loading - the mechanostat. More recently, Frost provides evidence for genetically determined stress thresholds for bone reabsorption, maintenance and remodelling of bone. ${ }^{10}$ Here, we propose a design process for FES bone health therapies that involves calculating the 3D distribution of microstress in the bone of interest - here we use the tibia of a paraplegic subject. A beam model of the tibia, scaled to the subject, will be used. This model will then be used to determine the regions of the tibia where osteogenesis is expected when using a FES bone health therapy - for example, FES rowing.

\section{Material and Methods}

Here we illustrate the design process using our RowStim(III) system with 4 channels of electrical stimulation (Quads + Hamstrings) as described in [2]. The rowing machine used was based on the fixed stretcher Concept 2 model (D) ergometer.The FES rowing subject RG and co-author was $57 \mathrm{yrs}, 75 \mathrm{~kg}$ male, $1.72 \mathrm{~m}$ height with a T4 AIS (A) injury sustained 11 years previously. The data was acquired in 2012 and RG started FES rowing in 2003 and has maintained 2-3 30 min sessions per week since then. Following an initial 10 minute warm-up the subject was asked to row as hard as he could for approximately 20 strokes. The trials analysed had an indicated mean power of $60 \mathrm{~W}$ on the Concept 2 display. 


\section{A Design Method for FES Bone Health Therapy in SCI}

Eur J Transl Myol 26 (4): 297-300

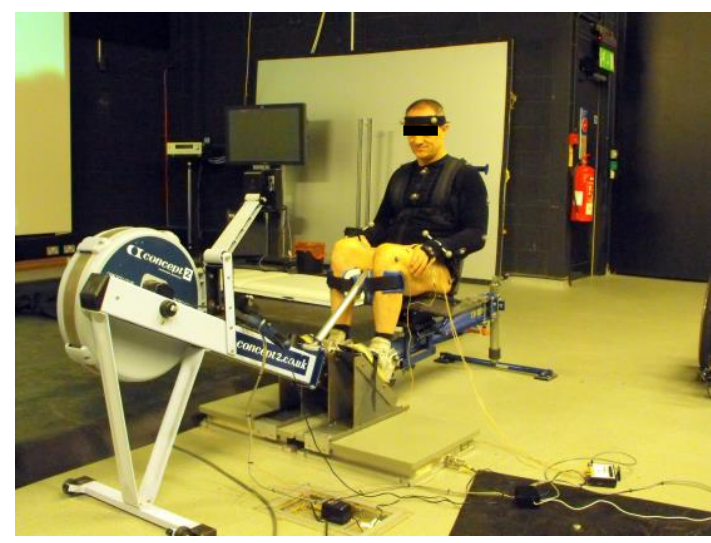

a

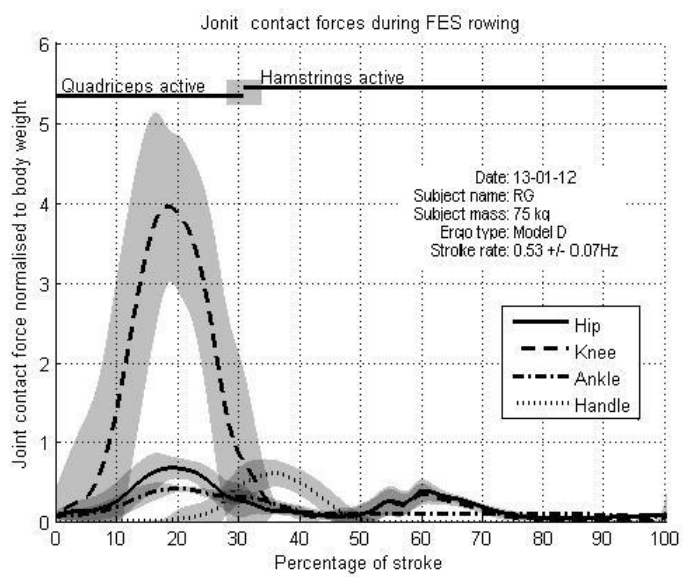

b

Fig .1 (a) Experimental set up used for FES Rowing. (b) Joint contact forces at the hip, knee and ankle joints obtained using BoB motion analysis inverse dynamics package www.marlbrook.com

The handle forces were measured using an in-line strain gauge transducer. The normal foot stretchers were replaced by cantilevers attached to two floor mounted OR6-7 force plates as shown in Fig 1(a). Kinematic and force data was acquired using a Vicon12 MX camera system running Nexus software.

The next step was to develop a beam model scaled to the tibial dimensions of RG. Stress distribution was calculated across the tibial bone model, assuming the tibia was fixed at the knee joint and forces were applied at the distal end of the tibia (i.e. a cantilever). ${ }^{8}$ Cross sectional measurements of the tibia were obtained from the literature. ${ }^{6}$ Capozza et al (2008). ${ }^{6}$ calculated, the periosteal perimeter, cortical thickness and second moment of inertia (in the longitudinal, lateral-medial and anterior-posterior axis) from $18 \mathrm{pQCT}$ scans of the entire tibia at intervals equivalent to $5 \%$ of the bone's length. This data was combined with lateral, compressive and bending forces obtained from FES rowing, to calculate the stress along the tibial bone. The perimeter of each pQCT slice was used to calculate the

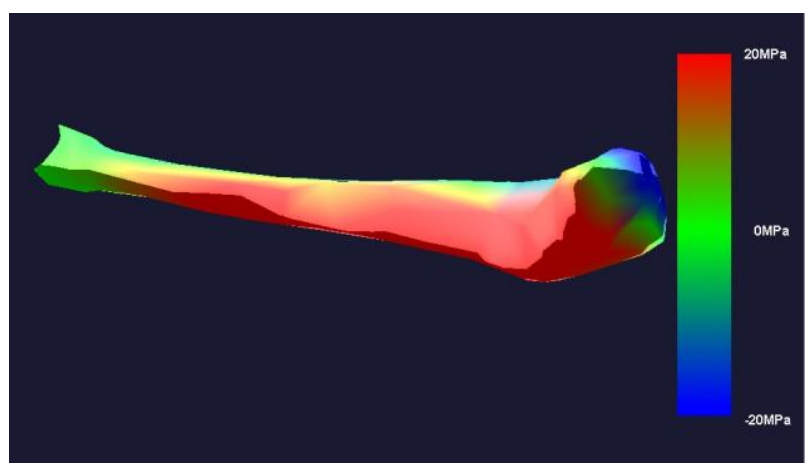

Fig 2. Stress distribution continuum along the tibial bone obtained from lateral, compressive and bending forces measured during 4-Ch FES rowing at a point in time. This figure corresponds to a single screen shot taken from a movie at the point of peak force from one perspective. Colours represent the stress values shown bv the colour bar on the right side of

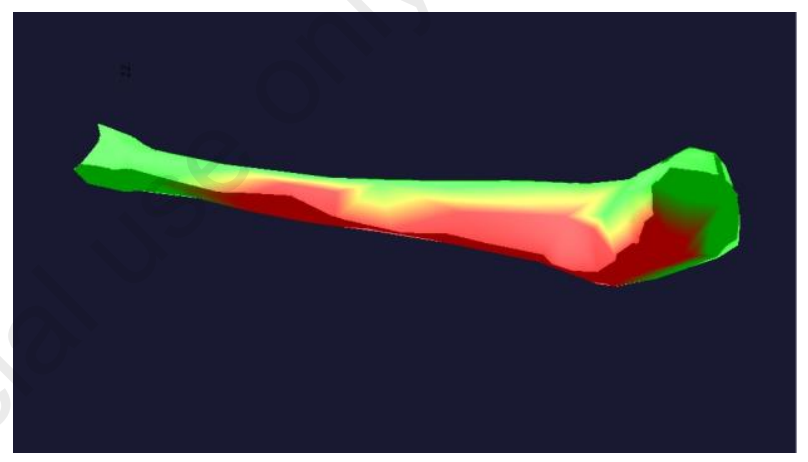

Fig 3. Showing regions of the tibia (red) that are above the Frost MESm 20 MPa threshold for the 4-Ch FES case (quadriceps only during the drive phase).

external radius. Using the values for cortical thickness the internal radius was calculated. The area of each section was obtained by assuming a hollow cylinder. To calculate the total stress we used standard moment and stress equations, ${ }^{8}$ i.e.

$$
\begin{gathered}
\sigma_{T}=\sigma_{\text {comp }} \pm \sigma_{\text {lat }} \pm \sigma_{\text {bend }} \\
\sigma_{T}=\frac{F_{\text {comp }}}{A} \pm \frac{M_{\text {lat }} r_{\text {out }}}{I_{L M}} \pm \frac{M_{\text {bend }} r_{\text {out }}}{I_{P A}} \\
M_{\text {lat }}=l * F_{\text {lat }} \text { and } M_{\text {bend }}=l * F_{\text {bend }}
\end{gathered}
$$

Where $r_{\text {out }}$ is the outer radius, $\mathrm{I}_{\mathrm{LM}}$ and $\mathrm{I}_{\mathrm{PA}}$ are the second moment of inertia in the lateral-medial and posterioranterior axes respectively and $l$ is the length of the tibia. 


\section{A Design Method for FES Bone Health Therapy in SCI}

Eur J Transl Myol 26 (4): 297-300

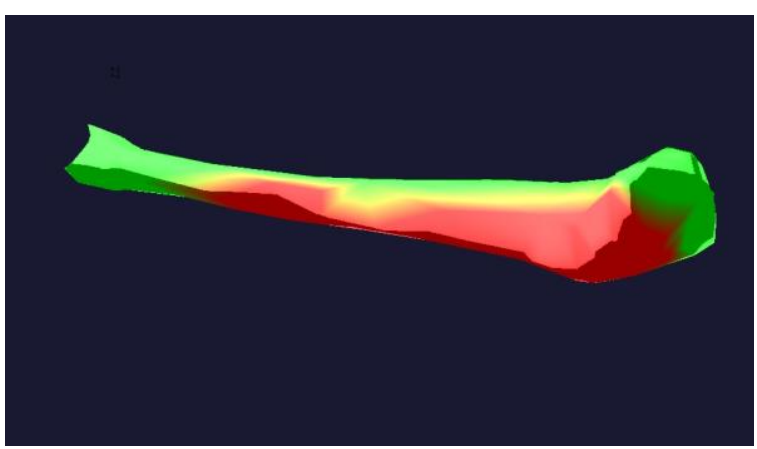

Fig 4. Showing the regions of the tibia (red) that are above the Frost MESm $20 \mathrm{MPa}$ threshold when the ankle plantar flexors are included in the drive phase of FES rowing (8-Ch FES).

\section{Results and Discussion}

We then applied the Frost threshold stress MESm, ${ }^{10}$ i.e. bone's genetically determined modeling threshold strain range, in and above which modeling usually turns on to strengthen a bone. Where MESm, 1000-1500 microstrain; $\sim 20 \mathrm{MPa}$, or $\sim 2 \mathrm{~kg} / \mathrm{mm}^{2}$. Applying the threshold at $20 \mathrm{MPa}$ can be seen in Figure 3. The fringing occurring at the edge of the threshold is an artefact due to the graphical colour interpolation and has no physical significance. The biomechanical calculation of joint contact forces, as shown in figure 1(b), exceed the 1.5 times body weight threshold proposed in Dudley-Javoroski S, Shields RK (2008). ${ }^{4}$ However, these plots not indicate how the loading is distributed throughout the bones such that regions of osteogenesis can be predicted. The 3D stress analysis allows detailed visualization of the stress applied to the tibia. Figure 2 is a typical single frame taken from a movie at the point of peak force and viewed from one perspective. Matlab software was written to enable 3D rotation to facilitate visual analysis. In this example, it is revealed that in no regions are unsafe levels of stress applied and that the Frost MESm threshold is exceeded in an extensive region of the tibia where one might expect osteogenesis. It also reveals regions that are under stressed such as the distal region and the posterior plateau. NB: here the cross sectional tibial measurements were obtained from healthy subjects. To the author's knowledge there are no corresponding data available for the SCI tibia. It is therefore possible that some of these measurements such as the thickness of the cortical bone and moment of inertia, would change in SCI subjects - this is an area where further radiographic studies are required to determine a model SCI tibia. The analysis was based simple beam theory using a point load applied to the distal tibia constrained as a cantilever so deflections, forces and boundary conditions are approximations.

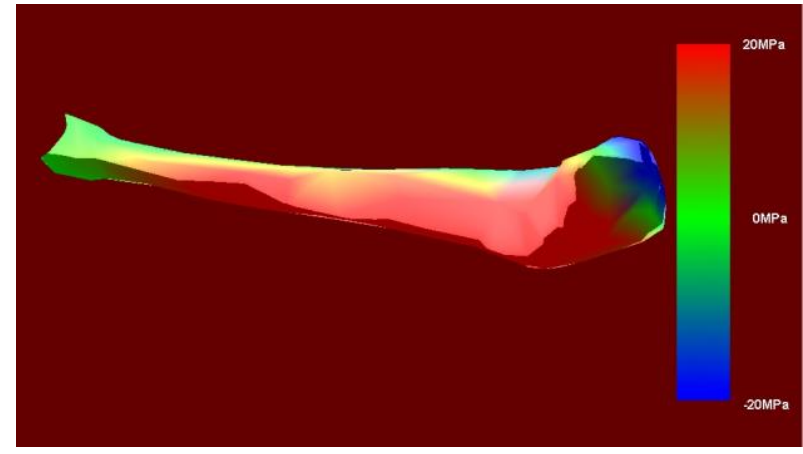

Fig 5. The regions of the tibia that are above $20 \mathrm{Mpa}$ threshold are shown in red. Here the gluteus maximus (biomechanically simulated) and quadriceps were both active bilaterally during in the drive phase of rowing. This suggests that supra-threshold stress extends further distally than for the 4-Ch case in figure 2.

The actual stress patterns illustrated can only be regarded as approximations but should be indicative of gross distributions and trends. However, at this stage the analysis presented here serves mainly to illustrate the proposed design technique. This provides a quantitative tool to generate hypotheses and further optimize the therapeutic application. For example, a number of experimental parameters could be manipulated. For example, in addition to the quadriceps, during the drive phase, it was expected that stimulating the ankle plantar flexors, in phase with the quadriceps during drive would extend distally the region that is above the MESm threshold 20MPa. However, in practice, figure 4 illustrates that this was not the case, with our beam model in which the ankle joint was simplified, however, the above threshold region around the condyle was extended, indicating that stresses in the distal tibia are similar the 4-Ch FES case and a little more widely distributed in the proximal region. Other parameters could then be experimentally explored in a stepwise design and development process. For example, electrically stimulating the hip extensors or changing the rowing style (e.g. the coordination between handle force and seat position during the drive phase) or the type of rowing ergometer used, e.g. floating versus fixed stretcher types. ${ }^{7}$ At each step in the design process the above stress analysis is performed and compared with alternatives to check progress towards a solution. Thus relatively few subjects would be involved in finding a solution that provides the best distribution of stress. This would support the follow on clinical trials, involving larger groups of subjects, to validate the predictions. The personalized beam model may be extended to other bones that are compromised by osteoporosis. The beam model is computationally very efficient providing results in the order seconds on a Windows i7 PC. The present beam model may 


\section{A Design Method for FES Bone Health Therapy in SCI}

Eur J Transl Myol 26 (4): 297-300

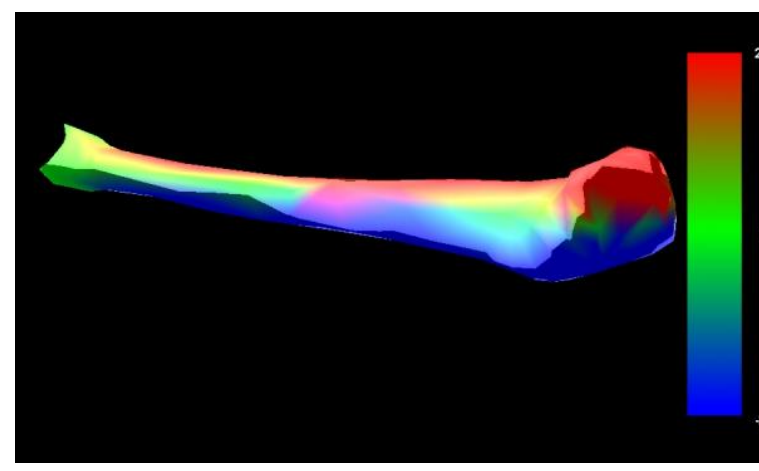

Fig 6. Stress distribution for the simulation of a "floating stretcher" type rowing machine where the subject is almost stationary. The supra-threshold regions are reduced compared with fixed stretch design.

underestimate the ankle region, due to the tibia, ankle joint and foot being represented by a single cantilever this we plan to improve. The stress distribution modelling may be further extended, with greatly increased computation time, by using a finite-element model derived from high resolution pQCT scans. The stress distribution model may be improved by adjusting thresholds that better predict actual clinical scan data and further extended by including a porosity stage that may provide a deeper understanding of the basic mechano-transduction, poroelastic fluid low and cellular signaling. ${ }^{9}$ The proposed technique may also be extended using simulated force data derived from biomechanical models of the therapeutic activity - in this case it may even be possible to design and evaluate a candidate FES bone therapy in-silico prior to clinical trials. This is illustrated in Figure 5 where the bi-lateral force actions of the gluteus maximus muscles were biomechanically simulated and added to the quadriceps actions during the drive phase. This produced a slightly wider distribution of stresses above $20 \mathrm{Mpa}$. These modest changes due to additional extensors. To demonstrate a more dramatic change in distribution we simulates a floating stretcher type rower by putting the acceleration of the center of mass of the upper body to zero (Figure 6). ${ }^{7}$ This produced a much reduced distribution of supra threshold stress.

In conclusion, the approach appears to offer a quantitative design tool and method to assess potential bone therapy benefits for FES assisted exercise activity.

\section{Author's Contribution}

All authors contributed equally.

\section{Acknowledgments}

Research supported by Department of Bio-Engineering at the University of Reading with funding from EPSRC (UK), Grant Ref: EP/L02019X/1.

\section{Conflict of Interest}

The authors declare no conflict of interests.

\section{Corresponding Author}

Brian Andrews, Bio-Engineering, University of Reading and The Nuffield Department of Surgical Sciences, Oxford, UK E-mail: brianandrews55@hotmail.com

\section{E-mail of coAuthors}

James Shippen: aa2388@ coventry.ac.uk Monica Armengol: m.armengol@reading.ac.uk William Harwin: w.s.harwin@ reading.ac.uk William Holderbaum: w.holderbaum@ reading.ac.uk Robin Gibbons: robin.gibbons15@gmail.com

\section{References}

1. Krause JS, Carter RE, Pickelsimer EE, Wilson D. A prospective study of health and risk of mortality after spinal cord injury. Arch Phys Med Rehabil 2008;89:1482-91.

2. Hettinga D, Andrews BJ. Feasibility of FES Rowing for High Energy Training and Sport, Neuromodulation 2007;10:1-7 .

3. Worthen LC, Kim M, Kautz SA, et al. Key characteristics of walking correlate with bone density in individuals with chronic stroke, J Rehab Res Dev 2005:42:761-8.

4. Dudley-Javoroski S, Shields RK. Asymmetric bone adaptations to soleus mechanical loading after SCI, J Musculoskel Neuronal Interact 2008;8:227-38.

5. Dudley-Javoroski S, Saha PK, Liang G, et al. High dose compressive loads attenuate bone mineral loss in humans with SCI, Osteoporosis Int 2011; doi:10.1007/s00198-011-1879-4.

6. Capozza RF, Cointry GR. Structural analysis of the human tibia by tomographic (pQCT) serial scans. Journal of Anatomy 2010;216:470-81.

7. Andrews BJ, Shippen J, Gibbons R, May B, Wheeler ?. FES Rowing Biomechanics: Fixed and floating stretcher ergometers. Proceedings of IFESS 2012;3-6.

8. Shigley J Mechanical Engineering Design, p44, International Edition, pub McGraw Hill, 1986, ISBN 0-07-100292-8.

9. Pereira AF, Shefelbine SJ. The influence of load repetition in bone mechanotransduction using poroelastic finite-element models: the impact of permeability, Biomech Model Mechanobiol 2014;13:215-25 doi10.1007/s10237-013-0498-8.

10. Frost $H$. The Utah Paradigm of Skeletal Physiology. Athens: ISMNI, 2003.

11. Eser P, Frotzler Y, Wick L, Knecht H, Denoth J, Schiessl H. Relationships between the duration of paralysis and bone structure: a QCT study of spinal cord injured individuals, Bone 2004;34:869880 . 